\title{
COMMENT
}

Check for updates

\section{Pulling at the heart: COVID-19, race/ethnicity and ongoing disparities}

\author{
Peter Chin-Hong ${ }^{1}$, Kevin M. Alexander (1) ${ }^{2}$, Norrisa Haynes ${ }^{3}$, Michelle A. Albert ${ }^{4 凶}$ \\ and The Association of Black Cardiologists*
}

Social determinants of health in the African diaspora drive the lack of disease testing, increased prevalence of comorbid disease and reduced access to drugs, resulting in disproportionately higher COVID-19-related mortality among Black individuals than the rest of the population. We urge decisive attention to and action against ethnicity-based inequities that undermine cardiovascular health.

The COVID-19 pandemic presents an opportunity to decisively address racebased or ethnicitybased inequities that undermine cardiovascular health

\begin{abstract}
${ }^{1}$ Division of Infectious Diseases, University of California, San Francisco, CA, USA.
\end{abstract}

${ }^{2}$ Division of Cardiology, Stanford University, Palo Alto, CA, USA.

${ }^{3}$ Division of Cardiology, University of Pennsylvania, Philadelphia, PA, USA.

${ }^{4}$ Center for the Study of Adversity and Cardiovascular Disease (NURTURE Center) Division of Cardiology, University of California San Francisco, CA, USA ${ }^{*} A$ list of authors and their affiliations appears at the end of the paper

凶e-mail:michelle.albert@ ucsf.edu

https://doi.org/10.1038 s41569-020-0416-6

\section{Epidemiology and social determinants}

The coronavirus disease 2019 (COVID-19) pandemic continues to ravage many communities worldwide, but the effect of COVID-19 on morbidity and mortality in the African diaspora, which includes Black individuals living in Brazil, the UK and the USA, is particularly devastating. African American individuals are over-represented in COVID-19 mortality statistics. COVID-19 contributed to 61.6 deaths (per 100,000 deaths) among Black individuals in the USA, compared with 28.2 deaths among Hispanic/Latinx individuals, 26.3 deaths among Asian individuals and 26.2 deaths among white individuals ${ }^{1}$. Black individuals in the UK, who are largely of Caribbean and African descent, have twice the rate of COVID-19-related deaths as would be expected on the basis of population prevalence'.

Numerous social determinants of health drive the detrimental effect of COVID-19 among ethnic minorities in Brazil, the UK and the USA, including socioeconomic status, access to health care, unemployment, high rate of employment in the service industry and inability to socially distance ${ }^{3}$. These factors result principally from discrimination and segregation that limit socioeconomic opportunity, promote household and neighbourhood overcrowding, and increase the burden of chronic medical conditions, including cardiovascular disease (CVD), cancer and asthma. Importantly, although socioeconomic status is tightly linked to health outcomes, Black individuals have diminishing returns on education and neighbourhood-level socioeconomic variables, and segregation persists regardless of income or education level ${ }^{4}$. Although individuals within the African diaspora are well known to have increased levels of pro-inflammatory markers compared with the rest of the population, which has been suggested to increase susceptibility to COVID-19-related mortality, this increased inflammation is largely influenced by body mass and social determinants of health ${ }^{5}$.

\section{Diagnosis}

The diagnosis of COVID-19 among ethnic minorities has its own set of challenges. Lack of access to diagnostic testing might exacerbate disparities among these populations, which can result in larger outbreaks if left unchecked. The US state and federal governments do not routinely categorize diagnostic testing data by ethnicity, making comparisons between ethnicities difficult. Some evidence suggests that access to testing for COVID-19 is dependent on residential address. An analysis of New York City Department of Health data found that over two-thirds of the 30 ZIP codes with the highest per-capita rates of testing were home to a higher proportion of white or wealthy individuals than the rest of the population, with similar findings in other US cities 6 . Factors for poor test uptake among minority populations include difficulty in accessing testing sites (such as locations poorly served by public transportation), federal public-private partnerships that prioritize profitability rather than need, high cost of tests, the requirement for mobile phone applications to access testing sites in some locations and the longstanding mistrust of health-care professionals. Targeted outreach to Black and Hispanic/Latinx individuals can address diagnostic challenges. For example, a coalition in San Francisco's Mission District (a densely populated neighbourhood with a large population of Hispanic/ Latinx individuals) was created by university and community partners. Over 4 days, $>4,000$ adults and children in one census tract were tested for COVID- 19. Approximately 95\% of those who tested positive were of Hispanic/Latinx background, despite making up only $58 \%$ of the population in the district. Moreover, $>50 \%$ of these individuals were asymptomatic at the time of testing and the majority were essential workers. As a result of this initiative, the city provided replacement wages, food and accommodation for those who needed to quarantine. 


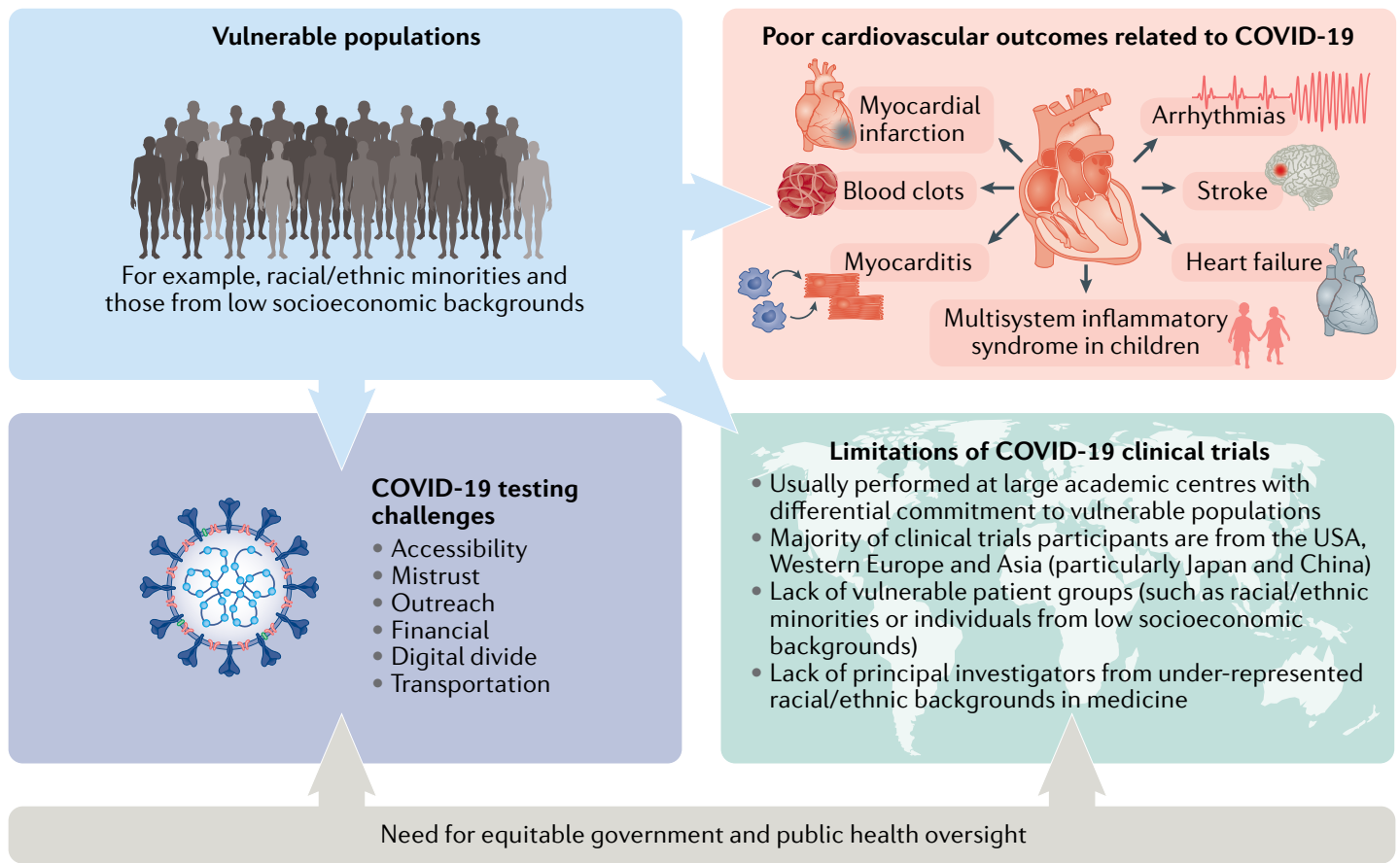

Fig. 1 | COVID-19 and racial/ethnic disparities. Summary of coronavirus disease 2019 (COVID-19)-related disparities in cardiovascular outcomes, rates of testing and clinical trial involvement among vulnerable populations. Publ. note: Springer Nature is neutral about jurisdictional claims in maps.

\section{Cardiovascular disease}

Unravelling the relationship between CVD and ethnicity is important to understand the high mortality in Black individuals with COVID-19. Cardiovascular conditions that disproportionately affect Black individuals, such as hypertension, are associated with worse COVID-19 outcomes ${ }^{7}$. Moreover, SARS-CoV-2, the virus that causes COVID-19, enters cells via angiotensin-converting enzyme 2 (ACE2), leading to ACE2 downregulation? This finding has raised questions on whether changes in the activity of the renin-angiotensin-aldosterone system (RAAS), such as with the use of antihypertension therapy with ACE inhibitors or angiotensin-receptor blockers, can have deleterious or beneficial effects on COVID-19 pathogenesis. Whether race/ethnicity-specific differences in the expression of ACE2 have an effect on susceptibility to COVID-19 requires evaluation.

A subset of patients with COVID-19 develop delayed-onset and severe symptoms induced by a hyperinflammatory response ${ }^{7}$. The potential cardiovascular sequelae of this immune dysregulation include myocarditis, decompensated heart failure, acute coronary syndrome and malignant arrhythmias. Furthermore, a rare multisystem inflammatory syndrome in children who tested positive for COVID-19 infection has been described. This syndrome resembles Kawasaki disease and can lead to severe complications, such as coronary aneurysms, thrombosis, multi-organ failure and shock. The collection of biomarker data from children of different racial/ethnic backgrounds will be important for the development of diagnostic tools, particularly for Black children, given the high COVID-19-related morbidity among Black adults.

Thromboembolism is a well-described complication of COVID-19. Thromboembolism incidence varies by race/ethnicity; African American individuals have a $30-60 \%$ higher incidence of thromboembolism than white individuals, which might be related to increased susceptibility to endothelial injury, increased hypercoagulability and higher rates of obesity and chronic inflammation ${ }^{8}$. Although data on the effect of race/ ethnicity on COVID-19-related thromboembolism are scarce, most COVID-19-related deaths are associated with cardiovascular causes. Given the cardiovascular risk factor profile of Black individuals ${ }^{8}$, catastrophic thromboembolic events might be more likely in Black individuals with COVID-19 than in white individuals with COVID-19. Furthermore, COVID-19 seems also to be associated with a higher incidence of stroke. African American individuals have a twofold to fourfold higher prevalence of stroke than white individuals, in part owing to a higher prevalence of hypertension ${ }^{8}$.

\section{Therapeutics}

To date, no drugs have been approved by the FDA for the treatment of COVID-19. Before the emergency-use authorization by the FDA of the moderately effective antiviral agent remdesivir, the only access to the most promising therapies for COVID-19 was through investigational drug trials. Pharmaceutical industry-sponsored or NIH-funded randomized clinical trials are typically performed at large academic centres with a regulatory and investigational pharmacy focus, but with differential commitment to vulnerable populations. In the ACTT-1 trial ${ }^{9}$, which evaluated the safety and efficacy of remdesivir in patients with COVID-19, very few of the sites at which patients were enrolled were minority-serving hospitals (defined as hospitals with twice as many Black or Hispanic/Latinx patients as expected on the basis of the percentage of those living 
in the region, or hospitals in which $>25 \%$ of patients in the intensive care unit were Black or Hispanic/Latinx). Furthermore, individuals from ethnic minority backgrounds were under-represented as both trial participants in the majority of medical studies and as principal investigators of trials, reflecting the dearth of diversity in academic medicine and science overall.

\section{Conclusions}

The interplay between COVID-19, race/ethnicity and medicine reflects the historical challenges faced by communities of colour worldwide (FIG. 1). Lessons from natural disasters could help to inform COVID-19 strategies, particularly concerning CVD. For example, in 2005, hurricane Katrina was responsible for at least a threefold increase in the incidence of myocardial infarction that persisted for many years, as well as an earlier age of onset of myocardial infarction, driven by increased rates of smoking, unemployment and loss of health-care insurance ${ }^{10}$. The incidence of post-traumatic stress disorder was also higher in Black individuals than in white individuals after the hurricane, owing in part to poorer mental health among Black individuals before the disaster, and less social support afterwards. CVD risk mitigation in Black and Hispanic/ Latinx individuals during the COVID-19 pandemic is paramount, owing to their increased risk of worsening heart failure, resultant inflammation and/or psychosocial stress compared with the rest of the population. The COVID-19 pandemic presents an opportunity to decisively address race-based or ethnicity-based inequities that undermine cardiovascular health.
1. APM Research Lab Staff. The color of coronavirus: COVID-19 deaths by race and ethnicity in the U.S. APM Research Lab https://www.apmresearchlab.org/covid/deaths-by-race (2020).

2. The OpenSAFELY Collaborative. OpenSAFELY: factors associated with COVID-19-related hospital death in the linked electronic health records of 17 million adult NHS patients. Preprint at medRxiv https://doi.org/10.1101/2020.05.06.20092999 (2020).

3. Haynes, N., Cooper, L. A. \& Albert, M. A. on behalf of the Association of Black Cardiologists. At the heart of the matter: unmasking and addressing the toll of COVID-19 on diverse populations. Circulation https://doi.org/10.1161/CIRCULATIONAHA. 120.048126 (2020)

4. Bucholz, E. M., Ma, S., Normand, S.-L. T. \& Krumholz, H. M. Race, socioeconomic status, and life expectancy after acute myocardial infarction. Circulation 132, 1338-1346 (2015)

5. Albert, M. A. et al. Race, ethnicity, and the efficacy of rosuvastatin in primary prevention: the Justification for the Use of Statins in Prevention: an Intervention Trial Evaluating Rosuvastatin (JUPITER) trial. Am. Heart J. 162, 106-114 (2011).

6. McMinn, S., Carlsen, A., Jaspers, B., Talbot, R. \& Adeline, S. In large Texas cities, access to coronavirus testing may depend on where you live. NPR https://www.npr.org/sections/health-shots/2020/05/27/ 862215848/across-texas-black-and-hispanic-neighborhoods-havefewer-coronavirus-testing-sit (2020).

7. Ruan, Q., Yang, K., Wang, W., Jiang, L. \& Song, J. Clinical predictors of mortality due to COVID-19 based on an analysis of data of 150 patients from Wuhan, China. Intensive Care Med. 46 846-848 (2020).

8. Albert, M. A. et al. Pulling at the heart: faces of COVID-19 survival and ongoing disparities in diverse populations [webinar]. Association of Black Cardiologists https://abcardio.org/covid19webinar/ (2020).

9. Beigel, J. H. et al. Remdesivir for the treatment of Covid-19preliminary report. N. Engl. J. Med. https://doi.org/10.1056/ NEJMoa2007764 (2020)

10. Jiao, Z. et al. Effect of Hurricane Katrina on incidence of acute myocardial infarction in New Orleans three years after the storm. Am. J. Cardiol. 109, 502-505 (2012).

\section{Acknowledgements}

This work was carried out on behalf of The Association of Black Cardiologists.

\section{Competing interests}

The authors declare no competing interests.

The Association of Black Cardiologists

Peter Chin-Hong', Kevin M. Alexander ${ }^{2}$, Norrisa Haynes ${ }^{3}$ and Michelle A. Albert ${ }^{4}$ 